\title{
Worldwide Recommendations and Therapies for Multiple Sclerosis: Are They Safe in the COVID-19 Pandemic Period?
}

\author{
Francesco Ferrara ${ }^{1}$ (D) $\cdot$ Francesco De Rosa ${ }^{2} \cdot$ Antonio Vitiello $^{1}$ \\ Accepted: 20 June 2020 / Published online: 26 June 2020 \\ (C) Springer Nature Switzerland AG 2020 \\ Keywords SARS-CoV-2 $\cdot$ Immunomodulating $\cdot$ Sclerosis $\cdot$ Depletive drugs $\cdot$ Myelin
}

\section{Dear Editor,}

SARS-CoV-2 infection has spread rapidly throughout the world causing a global pandemic and a health emergency with a few precedents in the human history. Some categories of people such as those with pre-existing comorbidities, the elderly, or people with chronic therapeutic treatments are at greater risk of infection and complications and can rapidly develop serious respiratory tract injuries. The host immune system is very important in the early stages of SARS-CoV-2 infection to combat viral replication. Patients with multiple sclerosis (MS) treated with immunomodulating pharmacological agents may have a higher risk of becoming infected with SARS-CoV-2 and associated complications. Proper management of the clinical pharmacological aspects of the MS patient is of paramount importance especially in this period of the COVID-19 pandemic. The data show that the MS patient has a higher risk of infection (about $+40 \%$ ) than the general population and the most frequent hospitalizations are caused by respiratory and urinary tract infections. This is why it can be said that the patient with MS is a fragile patient, and in this pandemic period, he or she needs to be closely monitored. Treatments for MS are immunomodulatory and include first-line drugs such as interferon beta (IFN- $\beta$ ), glatiramer, dimethylfumarate, and teriflunomide and second-line drugs such as fingolimod, natalizumab, ocrelizumab, alemtuzumab, and cladribine. Thanks to these treatments, it is possible to control the disease for many years, but these therapeutic agents can also lead to

This article is part of the Topical Collection on COVID-19

Francesco Ferrara

francesco.ferrara@uslumbria1.it

1 Usl Umbria 1, Perugia, Italy

2 Campania Region, Naples, Italy adverse reactions with serious effects and an increased risk of viral, bacterial, and fungal infections [1-4].

The safety profile of IFN- $\beta$ and glatiramer has been evaluated in numerous studies; the most frequent adverse reactions recorded are flu-like syndrome symptoms and injection site reaction. At the moment, they seem to be the safest drugs to avoid the risk of infection. Fingolimod can lead to serious adverse reactions, and the most common are urinary and lower respiratory tract infections. These reactions have been shown to be due to the reduction of lymphocytes in the blood. The use of teriflunomide and dimethylfumarate shows that these drugs lead to a slight reduction in white blood cell counts that could expose the patient to serious infectious risks. Adverse events of ocrelizumab concern allergic reactions due to infusion, itching, rash, hives, hypoglobulinemia, and lymphocytopenia associated with secondary infections. Based on the current literature, patients with MS being treated with these drugs should be closely monitored for any increased risk of infection associated with the drugs and the disease. Medications such as IFN- $\beta$ and glatiramer seem to be safer in this respect; ocrelizumab or cladribine seems to lead to a higher risk of infection than other drugs. At the moment, the guidelines of the various countries and scientific societies have not always coinciding positions on this delicate issue $[3,5]$.

In general, no country recommends stopping MS treatment. All countries agree that IFN- $\beta$ is the safest drug and can also protect against COVID-19 infection due to its antiviral properties. Treatment with glatiramer is also considered to be very safe. The USA offers the doctor greater freedom of choice as to whether or not to continue with ongoing therapy. The Food and Drug Administration simply divided the drugs according to risk. In general all recommendations consider that first-line drugs (teriflunomide and dimethylfumarate) should be continued and suspended in case of COVID-19 infection; in Germany and Canada, suspension is also recommended in case of severe lymphopenia. In Italy the position is 
more cautious with regard to teriflunomide, and the possible shift towards interferon therapy should be evaluated. Secondline therapy with fingolimod does not have a common point of view among the various countries; in Germany and Canada, it is recommended to continue the therapy by carefully assessing the risk; in Italy the suspension or switch to IFN- $\beta$ is assessed. Impoverished drugs (cladribine, ocrelizumab, and alemtuzumab) should be carefully evaluated. Treatment with natalizumab is considered safe in this pandemic period; in Italy there is more caution with immediate suspension in case of COVID-19positive patients [1].

SARS-Cov-2 has highlighted even more the issue of drug safety for MS, especially for new immunomodulatory drugs with higher potential risks of infection. Is it safe to continue therapy during the pandemic? Based on the evidence in the literature, the position of agencies in different countries, and the complexity of MS treatment, it is not recommended to discontinue MS therapy but to carefully assess the risk of infection.

Author Contributions The manuscript was written entirely by the authors, and all authors made an equal contribution in the development of the paper.

Compliance with Ethical Standards There are no sensitive data and no patients were recruited for this study. The document does not conflict with ethical legislation.
Conflict of Interest The authors declare that they have no conflict of interest.

\section{References}

1. MS Society medical advisors. MS treatments and COVID-19 coronavirus. https://www.mssociety.org.uk/about-ms/treatments-andtherapies/disease-modifying-therapies/covid-19-coronavirus-and-ms (Accessed 01 June 2020).

2. Guan WJ, Ni ZY, Hu Y, et al. Clinical characteristics of coronavirus disease 2019 in China. N Engl J Med. 2020. https://doi.org/10.1056/ NEJMoa2002032.

3. Zhang C, Shi L, Wang FS. Liver injury in COVID-19: management and challenges. Lancet Gastroenterol Hepatol. 2020;5:428-30. https://doi.org/10.1016/S2468-1253(20)30057-1.

4. Huang C, Wang Y, Li X, Ren L, Zhao J, Hu Y, et al. Clinical features of patients infected with 2019 novel coronavirus in Wuhan, China [published correction appears in Lancet. 2020 Jan 30;]. Lancet. 2020;395(10223):497-506. https://doi.org/10.1016/S01406736(20)30183-5.

5. D'Amico E, Leone C, Caserta C, et al. Oral drugs in multiple sclerosis therapy: an overview and a critical appraisal. Expert Rev Neurother. 2015;15(7):803-24. https://doi.org/10.1586/14737175. 2015.1058162 .

Publisher's Note Springer Nature remains neutral with regard to jurisdictional claims in published maps and institutional affiliations. 